Gynäkologische Endokrinologie 2016 14:231-232 DOI 10.1007/s10304-016-0098-7

(c) Springer-Verlag Berlin Heidelberg 2016

CrossMark

\section{W. G. Rossmanith' · C. Schöfl ${ }^{2}$ T. Strowitzki}

'Frauenklinik Baden-Baden Balg, Klinikum Mittelbaden gGmbH, Baden-Baden, Deutschland

${ }^{2}$ Endokrinologie im Zentrum, Bamberg, Deutschland

${ }^{3}$ Abteilung für Gynäkologische Endokrinologie und Fertilitätsstörungen, Universitäts-Frauenklinik, Universitätsklinikum Heidelberg, Heidelberg, Deutschland

\title{
Die zentralnervöse Steuerung menschlicher Reproduktion verstehen
}

\section{Ein langer Weg für die Neuroendokrinologie}

Die gynäkologische Neuroendokrinologie - eine faszinierende Wissenschaft, die sich mit den zentralnervösen Steuerungsvorgängen besonders in der Regulation menschlicher Reproduktion beschäftigt. Die vielfältigen Ergebnisse der letzten Jahrzehnte zu wichten, zu bündeln und vor allem in einen klinischen Kontext zu bringen, hat sich dieses Leitthema von Gynäkologische Endokrinologie zum Ziel gemacht. Es soll vor allem unseren gegenwärtigen Wissensstand beleuchten und in einen klinischen Kontext bringen.

\section{Woher kommt unser Wissen um die Neuroendokrinologie?}

Erste Vorstellungen von der neurohumoralen Regulation der menschlichen Reproduktion reichen bis zum Beginn des vergangenen Jahrhunderts zurück. Im Jahr 1905 legte Scott erste gedankliche Fundamente für allgemeine Prinzipien einer neuroendokrinen Steuerung mit seinen Worten: „Wenn Erregung die Nervenendigungen erreicht, so führt dies meiner Meinung nach zur Entladung einiger Nervenspeicher." Damit postulierte er eine Sekretion aus Nervenzellen auf die Zunahme zellulärer Aktivität hin. Zwei Jahrzehnte später schlug Smith vor, dass die Gonaden nicht eigenständig funktionieren, sondern durch zentralnervöse Strukturen wie die Hypophyse kontrolliert werden. Hohlweg und Junkmann vermuteten dann 1932, dass Steroide aus dem

Ovar die Gonadotropinsekretion aus der Hypophyse durch Rückkoppelung beeinflussen. Erstmals wurde dadurch der endogene humorale Regelkreis aus einem neuralen Steuerungsorgan und dem nachgeordneten Erfolgsorgan aufgezeichnet. Harris und Green schrieben 1948, dass der Hypophysenvorderlappen durch eine neurohumorale Sekretion aus übergeordneten Hirnstrukturen wie dem Hypothalamus gesteuert werde. Ihre Vorstellungen stützten sich auf die Entdeckung eines Portalkreislaufs im Hypophysenstiel. Erstmals konnte 1960 McCann in hypothalamischen Extrakten eine gonadotropinsezernierende Aktivität nachweisen. Damit konnte er belegen, dass die Freisetzung der hypophysären Gonadotropine luteinisierendes Hormon (LH) und follikelstimulierendes Hormon (FSH) einer hypothalamischen Kontrolle unterliegt.

\section{》) Die Charakterisierung von GnRH war ein Meilenstein der neuroendokrinologischen Forschung}

Einen Meilenstein in der Neuroendokrinologie erreichten Schally und Guillemin 1971 mit der Reindarstellung und Synthese eines hypothalamischen Dekapeptids, das die Gonadotropine freisetzt. Die Charakterisierung des Gonadotropin-Releasing-Hormons (GnRH) be- stätigte die Hypothese von neuronalen Kerngebieten als Schaltzentralen humoraler Regulation. Mit dem Nachweis episodischer elektrischer Aktivität in hypothalamischen Zellverbänden gelang Knobil und Wildt in den 1980er-Jahren zum ersten Mal der Nachweis, dass episodische Sekretionsmuster zur Aufrechterhaltung neurohumoraler Aktivität notwendig sind und dass zeitgleich mit elektrophysiologischen Veränderungen die intermittierende Sekretion von hypophysären Hormonen in das Serum abläuft. Nur eine diskontinuierliche oder episodische hypothalamische GnRH-Freisetzung kann physiologische Gonadotropinspiegel aufrechterhalten. Das Prinzip der episodischen Freisetzung von neuroendokrinen Stimulationshormonen gilt als Grundprinzip des physiologischen Funktionierens eines komplexen Apparats.

\section{Wo stehen wir heute?}

Dass sich die wegweisenden Entdeckungen auf dem Gebiet der Neuroendokrinologie über einen so langen Zeitraum erstrecken, wird dadurch erklärt, dass sich Untersuchungen zur zentralnervösen Steuerung menschlicher Reproduktion sehr schwierig gestalten. Dies liegt zum einen an den anatomischen Bedingungen, die den Zugang zu den zentralnervösen Gebieten neuroendokriner Regulation aus naheliegenden Gründen beim Menschen stark erschweren. 
Zum andern ist die Sekretion hypothalamischer und hypophysärer Hormone intermittierend und nicht kontinuierlich, die Mengen sind oft winzig klein. Während sich in der Vergangenheit Untersuchungen zur Neuroendokrinologie eher mit der hierarchischen Regulation und den Rückkoppelungsmechanismen auf die zentralnervösen Gebiete durch Produkte aus peripheren endokrinen Zielorganen beschäftigten, zielen unsere Untersuchungen heute darauf ab, zelluläre und molekulare Mechanismen in einzelnen Zellen sowie Zellverbänden bei der neuroendokrinen Steuerung der Reproduktion sichtbar zu machen.

Erkenntnisse zur differenzierten Steuerung der GnRH-Freisetzung durch intrazelluläre Prozesse legen offen, dass zelluläre Einzelaktivität gebündelt wird, um eine koordinierte Massensekretion aus Neuronenverbänden zu erlauben. Zugleich zeichnen Untersuchungen zur neuronalen Expression von Botensubstanzen wie Neuropeptiden und -transmittern ein Bild auf molekularer Ebene für das komplizierte Netzwerk von Einzel- und Zellverbandsaktivitäten. Dadurch entsteht ein Modell, das ein kompliziertes Wechselspiel aus biochemischen, molekularen und zellulären Signalen widerspiegelt und zugleich Wechselaktionen von untereinander vernetzten spezialisierten Neuronen beschreibt. Die im Serum beobachteten Sekretionsprofile von reproduktiven Hormonen werden als Ergebnis vielfältiger Einflüsse auf zellulärer neuronaler Ebene gesehen. Gemeinsam mit Erkenntnissen zum geordneten Zusammenspiel zwischen neuroendokriner Stimulation und regulierenden Endorganen fügen sich diese vielfältigen Ergebnisse der zellulären und molekularen Physiologie und Pathologie $\mathrm{zu}$ unserem heutigen Bild von der zentralnervösen Regulation menschlicher Reproduktion.

\section{Die Beiträge dieses Schwer- punkts: eine Standort- bestimmung}

Erkenntnisse aus einem Jahrhundert der neuroendokrinologischen Untersuchungen sind verständlicherweise sehr zahlreich und durch ihre Vielzahl fast un- überschaubar geworden. Wir haben deshalb anerkannte Experten auf dem Gebiet der klinischen Neuroendokrinologie gebeten, für uns den gegenwärtigen Wissenstand um die zentralnervöse Regulation des Endokriniums zusammenzustellen und zugleich im klinischen Kontext $\mathrm{zu}$ wichten. Insbesondere soll Wert darauf gelegt werden, aus der vorliegenden Vielzahl der Ergebnisse die klinisch relevanten Daten zu ziehen und für das Verständnis der klinischen Physiologie und Pathologie zu bündeln. Es ist hier gut gelungen, komplexe Mechanismen in der Neuroendokrinologie nicht nur als Einzelwirkungen von Neuromodulatoren zu verdeutlichen. Vielmehr sollen die zusammengestellten Erkenntnisse in den bearbeiteten Themen zeigen, dass in einem biologischen System wie dem Menschen das ganze Funktionieren der Neuroendokrinologie auf einer Summe aus einzelnen zellulären, biochemischen, molekularen und elektrophysiologischen Aktionen beruht. Darüber hinaus stellt allerdings das Ganze, das koordinierte Gesamtfunktionieren der Neuroendokrinologie in der Reproduktion, mehr als die Summe der Einzelaktionen dar.

\section{Ein kurzer Ausblick: Wie geht es weiter?}

Wir haben gelernt, dass die Aktivität von Regelschleifen in der Neuroendokrinologie entscheidend von der gegenseitigen Beeinflussung der Einzelelemente innerhalb wesentlicher zentralnervöser Regulationsorgane abhängt. Dieses System von neuronalen Verschaltungen zeichnet sich durch multiple Wechselverbindungen von Schwingkreiselementen untereinander aus. Was die biochemische, molekulare und physiologische Grundlage für die Regulation von zentralen Pulsgeneratoren darstellt, ist noch nicht ausreichend belegt und wird unser Interesse aufrechterhalten. Mit unserem zunehmenden Verständnis und wachsenden Wissen um die Neuroendokrinologie der menschlichen Reproduktion kommt es möglicherweise auch zu einer Erkenntnis, die ein bekannter Naturwissenschaftler so zusammengefasst hat:
„Wieviel müssen wir wissen, um zu wissen, wie wenig wir wissen?"

Wir wünschen Ihnen bei der Lektüre dieser Ausgabe voll geballter Informationen und Erkenntnisse aus dem faszinierenden Bereich der Neuroendokrinologie viel Spaß - und gute neue Einsichten.

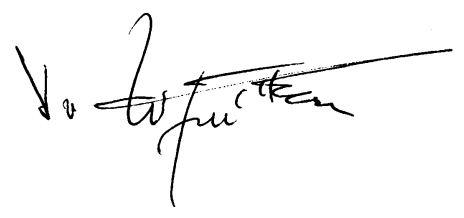

W. G. Rossmanith

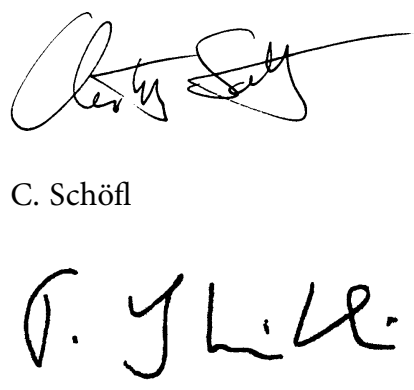

T. Strowitzki

\section{Korrespondenzadresse}

Prof. Dr. Dr. W. G. Rossmanith

Frauenklinik Baden-Baden Balg, Klinikum Mittelbaden $\mathrm{gGmbH}$

Balger Str. 50, 76532 Baden-Baden,

Deutschland

w.rossmanith@klinikum-mittelbaden.de

Interessenkonflikt. W. G. Rossmanith, C. Schöfl und T. Strowitzki geben an, dass kein Interessenkonflikt besteht. 